\title{
Revista Brasileira de Enfermagem REBEn \\ Compreendendo o significado da dor torácica isquêmica de pacientes admitidos na sala de emergência
}

\author{
Understanding the meanings of ischemic chest pain of patients \\ admitted in the emergency room \\ Comprendiendo el significado del dolor torácico por isquemia de \\ pacientes admitidos en la sala de emergencia
}

\section{Rachel Damaceno de Araújo}

Enfermeira. Aluna do Programa de PósGraduação em Enfermagem em Emergências da Faculdade de Enfermagem da Universidade de Santo Amaro, São Paulo, SP.

Isaac Rosa Marques

Enfermeiro. Mestre em Enfermagem. Professor Adjunto da Faculdade de Enfermagem da Universidade de Santo Amaro, São Paulo, SP. Orientador.

Artigo resultante da Monografia apresentada ao Curso de Pós-Graduação em Enfermagem em Emergências da Faculdade de Enfermagem da Universidade de Santo Amaro, em Março de 2007.

\section{RESUMO}

Os profissionais de enfermagem que atuam em serviços de emergência constantemente deparam-se com pacientes com dor torácica por isquemia. Este estudo objetivou compreender o significado deste tipo dor para o paciente durante a sua permanência na sala de emergência. Trata-se de um estudo de abordagem qualitativa realizado com dez pacientes admitidos em um serviço de emergência de um hospital privado localizado na zona sul da cidade de São Paulo. Os dados foram coletados por meio de entrevista semi-estruturada e analisados segundo a técnica de análise de conteúdo. Da análise decorreram os seguintes eixos temáticos: significado da dor torácica e sentimento no momento dos sintomas. Como resultados, pôde-se observar que o medo da morte, a preocupação com familiares são os significados mais importantes. Concluiu-se que os pacientes acometidos de dor torácica isquêmica necessitam apoio por parte da equipe de enfermagem no sentido de amenizar estes sentimentos.

Descritores: Enfermagem cardiovascular; Infarto do miocárdio; Dor aguda; Assistência de enfermagem.

\section{ABSTRACT}

Nursing professionals who work in emergency units are constantly facing patients with ischemic chest pain. This study aimed at understanding the meanings of patients with ischemic chest pain when they are in the emergency room. It is a study with qualitative approach that was carried with ten patients admitted in an emergency room in a private hospital in the south zone of São Paulo city. Data were collected through semi-structured interviews and analyzed according to content analysis approach. Resulting thematic axes were: meaning of chest pain and feelings when facing the symptoms. As results it was possible to observe the fear of death, family concerns were more significant. It was concluded that patients with ischemic chest pain need special support from the nursing team in order to decrease or diminish those feelings.

Descriptors: Cardiovascular nursing; Myocardial Infarction; Acute pain; Nursing care.

\section{RESUMEN}

Profesionales de enfermería que trabajan en servicios de urgencia constantemente de deparan con pacientes sufriendo de dolor torácico por isquemia. Este estudio tuvo como objetivo comprender el significado que el dolor tiene para el paciente durante su permanencia en la sala de emergencia. Tratase de un estudio de abordaje cualitativo realizado con diez pacientes admitidos en un servicio de emergencia de un hospital localizado en la zona sur del municipio de São Paulo. Los datos fueran recogidos por medio de entrevista semiestructurada y analizados según la técnica de análisis de contenido. De la análisis surgieron las siguientes clases temáticas: significado del dolor y sentimientos ocasionados por el dolor en lo momento de los síntomas. Como resultados, ha sido posible observar que el miedo de la muerte y la preocupación con la familia son los significados más importantes. Se hay concluido que los pacientes con dolor torácica por isquemia necesitan de apoyo del equipo de enfermería para amenizar estos sentimientos.

Descriptores: Enfermería cardiovascular; Infarto miocárdico; Dolor aguda; Atención de enfermería.

Araújo RD, Marques IR. Compreendendo o significado da dor torácica isquêmica de pacientes admitidos na sala de emergência. Rev Bras Enferm 2007 nov-dez; 60(6): 676-80.

\section{INTRODUÇÃO}

A dor torácica sugestiva de isquemia vem sendo um assunto de grande relevância a nível mundial, pois as estimativas confirmam, por exemplo, que cerca de 5 a $10 \%$ de todos os atendimentos realizados na Sala de Emergência anualmente nos Estados Unidos são direcionados a este tipo de paciente. Já em
Submissão: 25/08/2007

Aprovação: 15/10/2007 
nosso país, o Brasil, estima-se que a prevalência seja equivalente aos dados Norte-americanos, porém não existem números que comprovem tais informações. Acredita-se que anualmente, em nosso país, são realizados 4 milhões de atendimentos por dor torácica ${ }^{(1)}$. Nos EUA dentre os 8 milhões de atendimentos realizados anualmente, 1,2 milhões são diagnosticados como IAM, aproximadamente o mesmo percentual são classificados como angina instável e cerca de 2/3 acabam não confirmando uma causa cardíaca para os sintomas ${ }^{(1)}$.

Através das estimativas apresentadas pela sociedade Brasileira de Cardiologia, surge a questão: 0 que ocorre com esses "4 milhões" de pacientes no âmbito sentimental quando os mesmos estão na Sala de Emergência vivenciando a dor torácica?

Há relatos de pacientes que viveram a experiência do infarto, em que na maioria dos casos, o fato foi descrito como algo inexplicável, evidenciando dúvidas e inquietações ${ }^{(2)}$. Levando-se em consideração que este grupo era ciente de seu diagnóstico, houve o desejo de explorar quais sentimentos e experiências são vivenciadas por um grupo que está perante o desconhecido: a dor torácica. Desconhecido para quem a vivencia, desconhecido a princípio por parte também dos profissionais envolvidos no cuidar, já que a dor torácica pode estar associada a um IAM (presente em $70 \%$ dos casos de dor torácica) $)^{(3)}$, Angina instável, ou simplesmente não ter uma correlação com 0 sistema cardíaco(4).

A variedade das condições clínicas que se manifestam com a dor torácica faz com que haja uma tomada de decisões rápida com relação aos procedimentos, podendo assim aumentar o prognóstico para estes pacientes que apresentam tais sintomas ${ }^{(2)}$. Assim, algumas questões podem ser desencadeadas se a experiência vivenciado pelo grupo pesquisado pode ser analisada. Obter-se-á, por exemplo, informações sobre seus sentimentos, anseios e perspectivas o levará os profissionais enfermeiros a uma reflexão, como por exemplo, até que ponto a enfermagem está limitada no cuidado a este tipo de paciente?

Outra questão de pesquisa a ser respondida dentro deste contexto: o que o conhecimento do significado da dor torácica do paciente na sala de emergência pode contribuir para a melhora da assistência de enfermagem a estes pacientes?

Tendo por base este contexto, este estudo teve como objetivo descobrir o significado da dor torácica sugestiva de isquemia em pacientes quando estão na Sala de Emergência.

\section{REVISÃO DA LITERATURA}

\subsection{Dor torácica}

Uma das causas mais comuns de procura de assistência médica na sala de emergência, mas somente 10 a $15 \%$ dos pacientes que chegam com dor no peito apresentam IAM ${ }^{(4)}$. Conhecida popularmente como dor no peito, a dor torácica pode ser causada por várias condições clínicas que podem ser de contraturas musculares à infarto do miocárdio(4).

Uma das principais causas de morte em homens e mulheres são as "temidas" doenças cardiovasculares, mas somente $10 \%$ dos pacientes que procuram os serviços de emergência com dor torácica têm diagnóstico confirmado(5)

Geralmente os pacientes nem sempre admitem ter "dor" no peito. São comuns descrições como sensação de estrangulamento, constrição, peso, dor profunda, aperto, pressão, indigestão, queimação(6).

Quando a dor piora com a respiração na mudança de posição ou quando persiste por dois ou três dias em vários episódios, não é de natureza cardíaca. Geralmente quando se trata de dor torácica sugestiva de isquemia, o paciente não consegue apontar a o local da dor ${ }^{(6)}$.

A dor torácica sugestiva de isquemia pode manifestar-se no meio ou no lado esquerdo do tórax, sendo que essa sensação pode irradiar-se para o braço esquerdo, direito ou para ambos ou até para a região epigástrica. Há ainda pessoas que referem sentir dor na mandíbula confundindo-se com dor de dente(5).

\subsection{IAM - Infarto Agudo do Miocárdio}

O infarto acontece quando o tecido miocárdico é destruído em regiões do coração por onde o fluxo sanguíneo não é suficiente para sua irrigação devido a redução do fluxo sanguíneo coronariano(6), ou seja, o coração é uma bomba em constante funcionamento que envia sangue para todo 0 corpo. Sendo assim, contraindo e relaxando por volta de 100 mil vezes por dia. O coração necessita de ser suprido sempre com o sangue rico em oxigênio e nutrientes. Uma obstrução no trajeto sanguíneo pode fazer 0 coração diminuir seu ritmo de bombeamento sanguíneo(7).

Sendo assim, a falta de irrigação sanguínea no músculo cardíaco pode ocasionar lesões irreversíveis na parte posterior, anterior, inferior ou lateral dependendo da extensão do comprometimento( ${ }^{(7)}$. O diagnóstico é feito pelo ECG e de acordo com o resultado O IAM é classificado da seguinte maneira: IAM com supra desnivelamento de segmento ST (IAM com SST) e sem supra desnivelamento de segmento ST (IAM sem SST).

O infarto também pode ocorrer em pessoas que possuem coronárias normais. Isso pode ocorrer devido às coronárias apresentarem um espasmo, contraindo-se com violência, produzindo assim déficit parcial ou total na irrigação do músculo cardíaco(8).

Os sintomas são: dor ou forte pressão no peito, dor no peito refletindo nos ombros, braço esquerdo, pescoço e maxilar, dor abdominal, suor, palidez, falta de ar, perda temporária da consciência, sensação de morte iminente, náuseas e vômitos. E os fatores de risco são: histórico familiar de doença coronariana, idade (a partir dos 60 anos), colesterol alto, triglicérides elevado, hipertensão arterial, obesidade, diabetes, fumo, estresse e sedentarismo. A prevenção está relacionada com uma alimentação balanceada, manter 0 peso sob controle, praticar exercícios físicos, realizar exames de prevenção (Eletrocardiograma, Hemograma, glicose colesterol, etc) ${ }^{(7-9)}$.

Pacientes diabéticos são comumente acometidos pela doença arterial coronariana. Quando apresentam Diabetes Mellitus de longa duração, poderam ter infarto sem sentir dor, e nesses casos a sudoreses é fria, confundindo-se até com a hipoglicemia ${ }^{(5)}$.

\subsection{Angina}

Angina pectóris é a síndrome caracterizada por crises de dor ou sensação de pressão na região anterior do tórax. A causa vem do fornecimento insuficiente de sangue para as coronárias, reduzindo o oxigênio enviado para o miocárdio que acaba excedendo sua força em busca de oxigenação $0^{(5)}$. Se a angina não é adequadamente tratada, poderá evoluir para um ataque cardíaco(8).

Existem alguns tipos de angina que podem ser classificadas como:

- Angina instável (Angina pré infarto, angina crescente) - aumento progressivo na freqüência, intensidade e duração das crises anginosas.

- Angina estável Crônica - previsível, consistente, e acontece ao esforço e é aliviada pelo repouso

- Angina noturna - a dor ocorre à noite, geralmente durante o sono; Pode ser aliviada pela posição sentada. Comumente devida a insuficiência ventricular esquerda

\section{Angina de decúbito - angina enquanto deitado}

- Angina intratável ou refartária - angina grave e incapacitante

- Angina de Prinzmetal (variação: repouso) - tipo espontâneo de dor anginosa acompanhada de elevação do segmento ST no ECG. Pensa-se ser devido ao espasmo da artéria coronária associada ao alto risco de infarto

- Isquemia silenciosa - sinais objetivos de isquemia (como no teste de esforço), porém o paciente é assintomático(8).

Existem alguns fatores que podem desencadear a dor anginosa como; Esforço físico, provocando uma crise devida ao aumento das necessidades miocardicas de oxigênio; Exposição ao frio, causando vasoconstrição e elevação da pressão sanguínea, com o aumento da necessidade de oxigênio; Ingestão de uma refeição copiosa, aumentando o fluxo sanguíneo para a área mesentérica para a digestão, reduzindo a quantidade de sangue disponível 
para o coração (em um coração gravemente comprometido, o desvio do sangue para a digestão é o suficiente para iniciar a dor anginosa); estresse ou qualquer situação que leve a liberação de adrenalina e elevação da pressão arterial podem acelerar a freqüência cardíaca, aumentando assim a carga de trabalho do miocárdio. A detecção da angina requer o levantamento de um histórico cuidadoso; o tratamento eficaz começa com a educação do paciente $e^{(9)}$.

\subsubsection{Angina Estável}

É uma doença coronariana crônica que se caracteriza pela angina desencadeada aos esforços, que piora gradualmente e que melhora com 0 repouso ou com o uso de nitratos. Sua duração média é de 5 minutos, nunca maior que 10 minutos. Pode ser ocasionada também por desequilíbrio de demanda ( aumento do consumo de oxigênio)(9).

Dá-se o nome de fenômeno de warm-up quando um paciente desenvolve angina num determinado nível de esforço e após repetir o mesmo grau de esforço o episódio de dor não se repete ${ }^{(10)}$.

Os sinais e sintomas da angina estável são geralmente que começa frequentemente lenta e pode durar alguns minutos acompanhada de um grande mal-estar, palidez e sudorese, podendo ser causada por esforço físico e estresse.

\subsubsection{Angina instável}

Síndrome coronariana aguda que se caracteriza pela dor desencadeada em repouso ou aos mínimos esforços. A fisiopatologia é semelhante ao que ocorre no lam, com a diferença de que não existem sinais eletrocardiográficos, ou alterações das enzimas cardíacas e nem oclusão total do vaso corornariano ${ }^{(11)}$. A angina instável geralmente dura entre 10 e 30 minutos e é acompanhada de desconforto geral.

Os sinais e sintomas são: Dor persistente, geralmente não cessa com o fim do exercício físico por exemplo e pode ocorrer também com a perda da resposta da dor em relação aos medicamentos ${ }^{(11)}$.

Existe um grupo de pessoas que mesmo sem os sintomas devem estar atentas: os fumantes, os diabéticos, idosos com mais de 65 anos, hipertensos, indivíduos que apresentam colesterol com nível elevado e mulheres pós menopausa. Devem ser considerados os antecedentes familiares ${ }^{(8)}$.

\section{METODOLOGIA}

Este é um estudo de abordagem qualitativa cujo referencial teórico é o Interacionismo Simbólico. Considerando-se que a pesquisa foi realizada com um determinado grupo de pessoas que vivenciaram a dor torácica sugestiva de isquemia na Sala de emergência, acredita-se que o Interacionismo Simbólico contribuiu para que as experiências vivenciadas fossem transmitidas de forma que, através da interação entre pesquisador e pesquisado fosse possível definir situações e compreender significados ${ }^{(13,14)}$.

A amostra foi composta pela participação de 10 (dez) pacientes através de entrevista semi-estruturada (anexo 2) com a questão norteadora: Qual o significado que teve para o senhor(a) estar com dor torácica (peito) quando estava na Sala de Emergência? Também foi utilizado um formulário com dados sócio-demográficos como: idade, gênero, escolaridade, profissão, perfil de saúde antes da dor torácica, entre outros.

O projeto foi submetido a uma instituição hospitalar de médio porte e também junto à instituição de ensino (protocolo CEP 150/06) para consultar dados de pacientes que foram atendidos no serviço de emergência com 0 quadro de Síndrome Coronariana Aguda. A partir destes dados os pacientes foram contatados para participar do estudo, independentemente do local onde os mesmos se encontrassem no hospital.

Os pacientes que participaram do estudo consentiram por meio de assinatura do Termo de Consentimento Livre e Esclarecido, após estarem cientes de todas as informações contidas na Carta de Informação conforme resolução $196 / 96^{(14)}$. As entrevistas foram realizadas durante trinta minutos $e$ foram registradas em um gravador, sendo que posteriormente as informações foram transcritas e analisadas por meio da Técnica de Análise de Conteúdo ${ }^{(15)}$, afim de compor eixos temáticos emergidos das respostas dos pacientes.

\section{RESULTADOS E DISCUSSÃO}

Os dados foram coletados no período de Novembro de 2006 a Janeiro de 2007. Por se tratar de um estudo de abordagem qualitativa, esta composição amostral foi suficiente para obter a saturação de dados, o que permitiu a extração dos conceitos expostos como objetivos.

Dos 10 pacientes participantes, 8 tiveram diagnóstico médico de IAM e 2 de Angina Instável. Este resultado é conflitante se comparado com o que refere Bassan ${ }^{(8)}$, pois nota-se que $80 \%$ dos pacientes analisados tiveram IAM, já que em sua pesquisa somente 10 a 15\% tiveram diagnóstico confirmado.

A caracterização quanto ao gênero evidenciou predominância de pacientes do gênero feminino (7 sujeitos). Quanto à escolaridade, a maioria (80\%), tinha o Ensino Fundamental; enquanto 20\%, tinha o Ensino Médio.

Quanto à idade, a faixa etária de maior freqüência foi a de 50 a 59 anos (40\%). Em segundo lugar vem a faixa dos 70 a 79 anos de idade com $30 \%$ do percentual (Tabela 1).

Quanto ao estado civil, 50,0\% eram viúvos, 40,0\% casados, 10,0\% divorciados e $0,0 \%$ solteiros. Todos os participantes tinham filhos, sendo $70,0 \%$ com 3 filhos ou mais e $30,0 \%$ com 1 a 2 filhos.

Quanto à atividade de trabalho, 40,0\% eram aposentados, 40,0\% estavam empregados e $20,0 \%$ desempregados e quanto à atividade física $40 \%$ praticam algum ativiade ao menos uma vez por semana e $60 \%$ eram sedentários.

\section{Perfil de Risco para Doença Arterial Coronariana e IAM}

Conforme apresentado no Gráfico 1, dos fatores de risco conhecidos para Doença Arterial Coronariana, a HAS teve freqüência de 8 vezes, seguida pela História Familiar (3 vezes), Tabagismo (2 vezes), Etilismo e DM (1 vez).

O perfil da maioria dos pacientes sob estudo foi de mulheres com idade entre 50 e 59 anos, viúvas em sua maioria, com 3 ou mais filhos, aposentadas ou até mesmo empregadas (igualdade no percentual), hipertensas e que não praticam nenhuma atividade física.

A HAS é um dos fatores de risco mais preocupantes e que aumenta o risco de IAM. Segundo Colombo e Aguillar ${ }^{(16)}$, juntamente com o estresse e o tabagismo, este é um dos fatores mais presentes em pacientes que têm 0 primeiro episódio de IAM.

\section{Análise dos Dados Qualitativos}

A análise dos dados qualitativos resultou na categorização de dois eixos temáticos principais: significado da dor torácica e sentimento no momento dos sintomas. Sub-eixos decorrentes desta categorização incluem: Medo da Morte, Preocupação com Familiares, Sentimento de Comiseração e Percepção da Equipe de Atendimento.

A pessoa com dor torácica, em geral, é tomada de surpresa quando os sintomas aparecem ${ }^{(2)}$. Excetuando-se os pacientes portadores de Doença Arterial Coronariana e em uso de medicação sistemática(8).

A maioria dos pacientes procura o serviço de emergência em situação emergencial ou urgencial. Isto significa que a pessoa pode ou não estar passando por um processo patológico e o mesmo pode ou não estar relacionado com os sintomas atuais. Aintensidade dos sintomas causa na pessoa uma certa apreensão e inúmeros questionamentos que levam a organização mental e cognitiva para buscar o entendimento da situação.

Devido ainda à intensidade dos sintomas, a pessoa tende a ficar confusa e com medo. Dentre os medos, o medo da morte é o principal deles. Isto se dá pelo fato de a pessoa reconhecer que a intensidade dos sintomas é ameaçadora à manutenção da vida. E que as circunstâncias de sua ocorrência exigem que o paciente gaste energia para entender o que está acontecendo. 
Tabela 1. Faixas etárias dos participantes do estudo. São Paulo, 2006.

\begin{tabular}{lcc}
\hline Faixas Etárias (em anos) & $\mathbf{n}$ & $\%$ \\
\hline 50 a 59 & 4 & 40,0 \\
60 a 69 & 2 & 20,0 \\
70 a 79 & 3 & 30,0 \\
80 a 89 & 1 & 10,0 \\
\hline Total & 10 & 100,0 \\
\hline
\end{tabular}

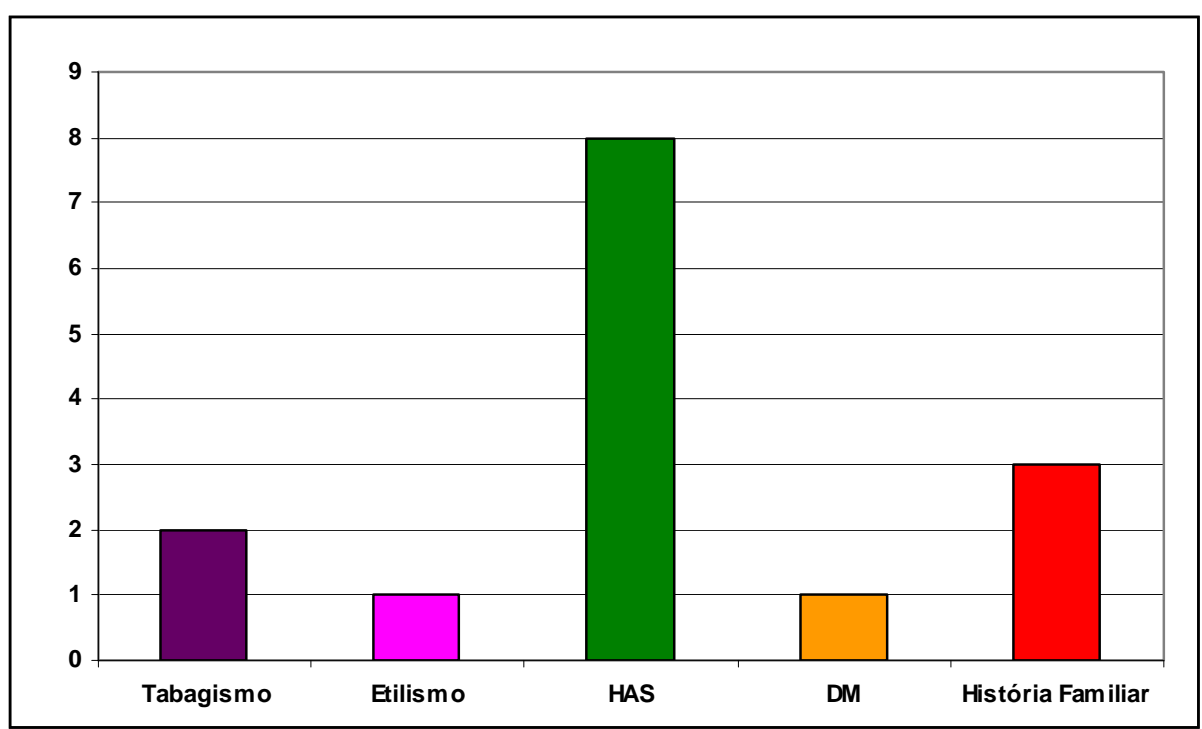

Gráfico 1. Freqüência dos fatores de risco para DAC. São Paulo, 2006.

\section{Medo da Morte}

O primeiro significado da presença da dor é a produção de um sentimento de morte eminente. A partir dos relatos apresentados a seguir, é possível verificar que a pessoa que passa pela experiência da dor torácica sente que está sob risco de morrer, conforme conteúdo dos discursos a seguir:

"Horrível, muito ruim, sensação de que ia morrer. Pedi a Deus que me ajudasse" (D1)

"Eu pensei que ia morrer de infarto, que eu estava gelada". (D2)

"Pensei que ia morrer. (D3)".

"Senti uma dor forte, pensei que ia acontecer o pior" (D4)

"Tive medo do castigo de Deus" (D5)

"Tive medo, talvez de morrer" (D11)

A partir dos discursos apresentados, foi possível perceber que os pacientes ao sentirem esta eminência da morte, reagem com medo e passam a fazer uso de estratégias de resolução de problemas. A primeira estratégia é buscar a ajuda superior (do divino) e também de buscar uma explicação para a dor, como por exemplo: atribuir à dor como se a mesma fosse um castigo divino (D5). É importante notar que o ser humano nesta hora também busca um alinhamento com as questões eminentemente espirituais. Isto vai desde uma simples prece até uma reflexão mais profunda sobre a sua existência.

A morte é considerada ainda uma tragédia pessoal, um castigo divino. Conhecer este significado a partir da pessoa que vivencia esta experiência, ajuda a entender a inquietação e o silêncio apresentados pelo paciente. Para os enfermeiros, é importante considerar o que o paciente sente, mesmo tendo que concentar-se nos aspectos técnicos peculaires envolvidos neste tipo de assistência. Tendo esta consciência, deve o enfermeiro também atender as necessidades psico-espirituais do paciente, aliviando os seus temores.

\section{Preocupação com Familiares}

O segundo significado é a preocupação com os familiares. Ao chegar no serviço de emergência, a pessoa normalmente é encaminhada para a sala de emergência. Neste ambiente inóspito e desconhecido, a pessoa sente-se isolada daquilo que é mais importante nesta hora: a família.

As pessoas com dor torácica isquêmica são, em geral, responsáveis por uma família e não apenas membros de uma família. Em geral, são estas pessoas as responsáveis pela manutenção financeira da família. Neste caso, a preocupação com os familiares é imediata. Juntando com o medo de morrer, emergem discursos como os apresentados as seguir:

"Então isso pra mim significou que eu não voltava mais, não ia ver mais minha família" (D4)

"Pedi a meu Deus: olha pra mim, olha pelo menos para os meus filhos, não deixe os meus filhos ficarem à toa" (D3).

"Fiqueipensando, com medo de morrer e deixar meus filhinhos pra trás"(D5)

"Pensei nos meus filhos". (D11)

Com base nestes discursos é possível evidenciar que a família tem um importante significado para a pessoa, pois gera neste momento um fator a mais de preocupação. Aligação com os familiares e o fato de que os mesmos possam ter algum tipo de dependência, a perspectiva futura sem a sua presença ou suporte, causa na pessoa ainda mais preocupação. Isto pode 
ser um fator que contribui para o aumento da descarga adrenérgica, podendo resultar em aumento do trabalho miocárdico e, consequentemente, aumento do consumo de oxigênio, gerando aumento da dor $(1,4,9)$.

O aumento tanto do consumo de oxigênio e da dor pode extender a área infartada e acarretar maiores complicações, como exemplos, as arritmias ventriculares, baixo débito cardíaco por falência ventriculare esquerda e/ou choque cardiogênico(4).

\section{Sentimento de Comiseração}

A comiseração é a explicitação do sentimento de que se está em miséria, que pede piedade, que se tenha compaixão. Este sentimento é perceptível neste momento. A pessoa procura alternativas que expliquem a causa da dor, buscando uma atenção para si. Os discursos apresentados a seguir contêm fragmentos deste conteúdo.

"Complicado.... o susto que eu tomei... eu não esperava que era um infarto mesmo..." (D2)

"O sentimento é péssimo, aí você pensa em tudo o que podia ter feito pra evitar"

"A dor é tão terrível que tem hora que você prefere morrer" (D9)

"Uma coisa não muito boa, que estava doente" (D8)

"Pensei que era um derrame, uma parada".

"A gente fica com medo de ficar torto, aleijado, dependendo dos outros"(D3)

"Fiqueicommedo de passar por tudo de novo, porquejáfiz angioplastia" (D10)

\section{Percepção da Equipe de Atendimento na Sala de Emergência}

"No Pronto Socorro muito carinho, porque às vezes dá desespero, elas vem e conversam, eu achei isso muito sensível". (D1)

"As enfermeiras até brincavam comigo pra ver se eu me acalmava um pouco". (D2)

"...me confortou ela conversar comigo". (D5)

"Me atenderam bem, com muito carinho". (D7)

É de extrema importância para a equipe que atua na sala de emergência dando maior ênfase ao profissional enfermeiro, que o profissional saiba como ele é visto pelo paciente no momento de sua angústia, no momento que recebe os cuidados durante 0 auge dos sintomas do IAM. Com esta percepção, o profissional pode dedicar algum tempo para assistir também os aspectos relacionados às necessidades psico-espirituais do paciente.

\section{CONCLUSÃO}

A partir dos dados coletados foi possível entender ou ampliar 0 conhecimento sobre o significado da dor torácica para o paciente que está na sala de emergência.

Os significados aprendidos foram: medo da morte, pelo fato de os pacientes desconhecerem o diagnóstico neste momento, e por sentirem a intensidade e a gravidade dos sintomas, sentem-se com a real possibilidade de morrerem. Outro eixo temático foi a preocupação com os familiares: os pacientes referiram que a ligação com os membros familiares e a relação de dependência que os mesmos tem em relação a si é um fator gerador de maior preocupação. 0 terceiro eixo temático é o sentimento de comiseração: a pessoa com dor torácica sugestiva de isquemia sente-se abatida e impotente diante deste quadro. Isto foi demonstrado nos discursos que revelaram a necessidade de compaixão e atenção. $O$ quarto eixo temático resultante foi a percepção da equipe que presta 0 atendimento. Neste sentido, os pacientes demonstraramse satisfeitos com 0 atendimento prestado, principalmente pela equipe de enfermagem em relação às necessidades psicológicas.

Conclui-se que o conhecimento obtido sobre os significados da dor pode contribuir para que os profissionais de enfermagem entendam melhor o que se passa com os pacientes no momento da ocorrência desta dor na sala de emergência. Estratégias para a amenização destes sentimentos deverão fazer parte de estudos futuros, evitando assim, que estes sentimentos influenciem ainda mais o quadro clínico do paciente.

\section{REFERÊNCIAS}

1. Bassan R, Pimenta L, Leães PE, Timerman A. Sociedade Brasileira de Cardiologia. I Diretriz de dor torácica na sala de emergência. Definição de graus de recomendação e níveis de evidência. Arq Bras Cardiol 2002; 76(supl II): 1-22.

2. Santos FLMM, Araújo TL. Vivendo o infarto: os significados da doença segundo a perspectiva do paciente. Rev Latino-am Enfermagem 2003; 11(6): 742-8.

3. Higa SEM, Atallah NA. Guias de medicina ambulatorial e hospitalar São Paulo (SP): Manole; 2004.

4. American Heart Association. Associação Interamericana do Coração. Suporte avançado de vida em cardiologia (Manual do ACLS). New York (NY): AHA; 2004.

5. Smeltzer S, Bare B. Brunner \& Suddarth - Tratado de enfermagem médico-cirúrgica. $8^{\mathrm{a}}$ ed. Rio de Janeiro (RJ): Guanabara-Koogan; 2002.

6. Eye GV. O ataque do coração. São Paulo; 2001. [citado em: 2007 abr 07]. Disponível em: URL:http://www.abcdasau de.com.br/artigo.php?300

7. Universidade Federal de Pernambuco. Angina. Recife; 2000 [citado em: 2007 abr 07]. Disponível em:URL:http:// www.ufpe.br/utihc/angina.htm

8. Bassan R. Unidade de dor torácica:uma forma moderna de manejo de pacientes com dor torácica na sala de emergência. Arq Bras Cardiol 2002; 79(2): 196-202.
9. Scofano M, Bassan R. Dor torácica na sala de emergência: a importância de uma abordagem sistematizada. Arq Bras Cardiol 2000; 74(1): 13-21.

10. Lopes,CA. Dor no peito, procure o médico. Rev Med Social Grupo 2007; 22(197): 26-7.

11. Fonseca ZA. Infarto do miocárdio: você conhece as causas? Belo Horizonte; 2005. [citado em 2007 abr 21]. Disponível em: URL: http://www. plantamed.com.br/div/infarto_do_miocar dio.htm

12. LoBiondo-Wood G, Haber J. Pesquisa em enfermagem - métodos, avaliação crítica e utilização. $4^{a}$. ed. Rio de Janeiro (RJ): Guanabara-Koogan; 2001.

13. Lopes CHA, Jorge MSB. Interacionismo Simbólico e a possibilidade para 0 cuidado interativo em enfermagem. Rev Esc Enferm USP 2005; 39(1): 103-8.

14. Ministério da Saúde. Conselho Nacional de Saúde. Diretrizes e Normas Regulamentadoras de Pesquisas Envolvendo Seres Humanos. [citado em: 2006 jul 18]. Disponível em: URL: http:/ /www.ufrgs.br/bioetica/res19696.htm

15. Bardin L. Análise de conteúdo. Lisboa.(POR): Edições 70; 1977.

16. Colombo RCR, Aguillar OM. Estilo de vida e fatores de risco de pacientes com primeiro episódio de infarto agudo do miocárdio. Rev Latino-am Enfermagem 1997; 5(2): 69-82. 